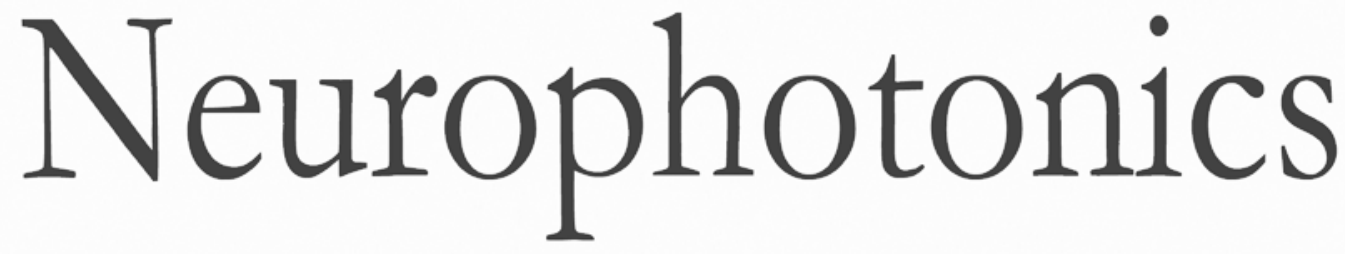

\title{
Spatial landscape of oxygen in and around microvasculature during epileptic events
}

Cong Zhang

Mohammad Moeini

Frédéric Lesage 


\section{Spatial landscape of oxygen in and around microvasculature during epileptic events}

\author{
Cong Zhang, ${ }^{\mathrm{a}, \mathrm{b}}$ Mohammad Moeini, ${ }^{\mathrm{a}, \mathrm{b}}$ and \\ Frédéric Lesage ${ }^{a, b, *}$ \\ ${ }^{a}$ École Polytechnique de Montréal, Department of Electrical \\ Engineering, Montreal, Quebec, Canada \\ bMontreal Heart Institute, Montreal, Quebec, Canada
}

\begin{abstract}
Measuring changes in cerebral oxygen in tissue microdomains during epilepsy is important to identify hypoxic potential. Here, using a custom-built two-photon microscopy system, we present microscopic measurements of oxygen partial pressure $\left(\mathrm{PO}_{2}\right)$ in cortical microvessels and tissue of anesthetized mice during 4-aminopyridine (4-AP)-induced epileptic seizures. Investigating epileptic events, we characterized the distribution of the "initial dip" in $\mathrm{PO}_{2}$ in arterioles, venules, and tissue near the 4-AP injection site. Our results reveal a correlation between the percent change in $\mathrm{PO}_{2}$ during the "initial dip" and the diameter of nearest arterioles and venules. ๑ 2017 Society of Photo-Optical Instrumentation Engineers (SPIE) [DOI: 10.1117/1.NPh.4.1 .010501]
\end{abstract}

Keywords: two-photon phosphorescence lifetime microscopy; oxygen partial pressure; epilepsy.

Paper 16064LR received Oct. 31, 2016; accepted for publication Feb. 17, 2017; published online Mar. 11, 2017.

\section{Introduction}

The global architecture of blood supply to the brain consists of a planar mesh of pial arteries and veins branching and diving into the cortex, to supply and drain blood ${ }^{1-3}$ in support of metabolic needs. A thorough understanding of the dynamics of oxygen supply and consumption in the cerebral cortex is important not only because neuronal activity relies on oxygen availability but also because a large number of functional imaging techniques (e.g., functional magnetic resonance ${ }^{4}$ and optical imaging of intrinsic signal ${ }^{5,6}$ ) rely on the changes in blood oxygenation to image brain function. Oxygen is transported to the brain by blood and delivered to tissue at the arteriolar and capillary level by diffusion. With increases in neural activity and metabolic consumption, the delivery of oxygen to tissue increases through changes in cerebral blood flow $(\mathrm{CBF})^{7,8}$ Under normal conditions, increases in blood flow slightly overshoot the requirement for tissue consumption, an overshoot sometimes argued to be neuroprotective. However, during excessive neuronal activity, as seen in epilepsy, supply might be insufficient.

*Address all correspondence to: Frédéric Lesage, E-mail: frederic.lesage@ polymtl.ca
Epilepsy is a common neurological disease characterized by recurrent unprovoked seizures, which result from abnormal and excessive neuronal activity in the brain. In both animal models and patients, the epileptic events can evoke drastic increases in $\mathrm{CBF}$ to meet the high metabolic demand caused by this intense neuronal activity. ${ }^{9-11}$ Measures of oxygen partial pressure $\left(\mathrm{PO}_{2}\right)$ in tissue during seizures have displayed an initial dip with various methods ${ }^{12,13}$ but the spatial landscape of this phenomenon has not been thoroughly documented. Recent data showed that most capillaries released little oxygen at baseline acting as an oxygen reserve that was recruited during increased neuronal activity. ${ }^{14}$ Whether this redistribution of the delivery of $\mathrm{PO}_{2}$ around and in capillaries during ictal events remains valid is unknown, and it is unclear how larger vessels modulate the spatial landscape of the $\mathrm{PO}_{2}$ distribution. In this study, we used twophoton microscopy (TPM) and the $\mathrm{O}_{2}$-sensitive phosphorescent dye PtP-C $343^{15}$ to measure $\mathrm{PO}_{2}$ in arterioles, venules, and tissue during epileptic events to characterize oxygen delivery during these periods of high demand.

\section{Materials and Methods}

\subsection{Animal Preparation}

A total of $N=11$ males C57/BL6 mice (postnatal 8 weeks old, 20- to 25-g weight) were used for this study, of which three died before data acquisitions due to procedures. Four mice were used for vascular $\mathrm{PO}_{2}$ measurements and four mice were used for tissue $\mathrm{PO}_{2}$ recordings. The Animal Research Ethics Committee of the Montreal Heart Institute approved all surgical procedures, which were performed according to the recommendations of the Canadian Council on Animal Care. All procedures were done according to the animal research: reporting of in vivo experiments guidelines (ARRIVE).

Animals were anesthetized with urethane (1 to $1.6 \mathrm{~g} / \mathrm{kg}$, intraperitoneal injection), tracheotomized, and maintained at constant body temperature $\left(37^{\circ} \mathrm{C}\right)$ with a controlled physiological monitoring system (Labeotech, Lachine, Canada). A moderate flow of ambient air lightly supplemented with oxygen was supplied next to the tracheotomy (10\% oxygen, $90 \%$ air, $1 \mathrm{~L} / \mathrm{min})$. The mice were then head-fixed with a stereotaxic device. After the injection of a local anesthetic (Xylocaine, subcutaneous, $0.2 \%$ ) under the scalp, a $\sim 2 \times 2 \mathrm{~mm}$ cranial window was opened over the right hemisphere to expose the somatosensory cortex and surrounding brain (anteroposterior: $-1.5 \mathrm{~mm}$ and dorsoventral: $+1.5 \mathrm{~mm}$ ). A small hole was drilled next to the cranial window for the injection of 4-AP. For tissue $\mathrm{PO}_{2}$ measurements, $\sim 1 \mu \mathrm{L}$ of the oxygen-sensitive dye (PtP-C343, $200 \mu \mathrm{M}$, synthetized based on published procedures $)^{16}$ was injected into the tissue and the cranial window was sealed with $1 \%$ agarose in artificial cerebrospinal fluid $(125 \mathrm{mM} \mathrm{NaCl}$, $10 \mathrm{mM}$ HEPES, $10 \mathrm{mM}$ glucose, $5 \mathrm{mM} \mathrm{KCl}, 1,5 \mathrm{mM} \mathrm{CaCl}_{2}$, and $1 \mathrm{mM} \mathrm{MgSO}_{4}$ ) using a $150-\mu \mathrm{m}$-thick microscope coverslip. For vascular measurements, the same dye was injected through the tail vein at 10 to $15 \mu \mathrm{M}$ initial concentration. A catheter in the femoral artery was used to monitor blood gases $\left(\mathrm{PCO}_{2}, 36\right.$ to $39 \mathrm{~mm} \mathrm{Hg}$ and $\mathrm{PO}_{2}, 110$ to $160 \mathrm{~mm} \mathrm{Hg}$ ). The average blood pressure ( 80 to $110 \mathrm{~mm} \mathrm{Hg}$ ) was measured using volumepressure recordings performed immediately prior to imaging. For each experiment, we labeled the blood plasma with fluorescein isothiocyanate conjugated with dextran (Sigma; $128 \mathrm{mM}$,

2329-423X/2017/\$25.00 (C) 2017 SPIE 
$200 \mu \mathrm{L}$ ) and used TPM to obtain a high-resolution structural image of the microvasculature. A larger image of the surface microvasculature $(\sim 2 \times 2 \mathrm{~mm})$ was also obtained, which was later used for easier tracing of the pial arterioles and venules.

\subsection{Epileptogenesis and Electrophysiology}

Epilepsy was induced by injecting the potassium channel blocker 4-AP (Sigma; $15 \mathrm{mM}, 0.5 \mu \mathrm{L}$ ) ${ }^{9}$ through a glass microelectrode using a syringe pump controller (UMP3, WPI) into a small hole with a depth of $\sim 300 \mu \mathrm{m}$ next to the cranial window at a rate of $100 \mu \mathrm{L} / \mathrm{min}$. Extracellular local field potentials (LFPs) were recorded with a tungsten electrode (impedance, $\sim 1$ to $2 \mathrm{M} \Omega$ ) lowered to a depth $\sim 300 \mu \mathrm{m}$ into the neocortex. The signal from the electrode was filtered by a bandpass filter (1 to $5000 \mathrm{~Hz}$ ), amplified with a microelectrode AC amplifier (model 1800, A-M system, Sequim, Washington), and digital filtered between 0.2 and $130 \mathrm{~Hz}^{13}$

\subsection{Two-Photon Microscope Setup, Acquisition, and Processing}

Measurements were collected on a custom-built time-domain two-photon phosphorescence lifetime microscope setup with $80 \mathrm{MHz}, 150 \mathrm{fs}$ pulses from a MaiTai-BB laser oscillator (840 nm, Newport Corporation, Irvine, California) with output going through an acousto-optic modulator (ConOptics) to adjust the gain for depth-dependent two-photon excitation intensity and gate the laser during lifetime imaging. Reflected light was collected by a water-immersion objective $(20 \times, 1.0 \mathrm{NA}$; Olympus) and then separated into two beams (phosphorescent photons and fluorescent photons) by dichroic mirrors. Phosphorescent light was passed through a filter centered at $680 \mathrm{~nm}$ and detected by a first photomultiplier tube (H7422, Hamamatsu, Photonics, Japan) and fluorescent light was filtered around center wavelength of $520 \mathrm{~nm}$ and detected by the second photomultiplier tube (R3896, Hamamatsu Photonics, Japan). Scanning and data recordings were controlled by customdesigned software written in MATLAB ${ }^{\circledR}$ (MathWorks, Natick, Massachusetts). Excitation pulses of $25 \mu$ s followed by $275 \mu \mathrm{s}$ lifetime recording were averaged 1000 times. Phosphorescence lifetime was fitted with a single-exponential function using a least-squares method. The lifetime was converted to $\mathrm{PO}_{2}$ using a calibration plot obtained in independent oxygen titration experiments of the same dye batch. ${ }^{15}$

For each animal, a picture of the craniotomy along with the real-time display of the surface vessels scanned was used to localize arterioles and venules. These and real-time imaging of two-dimensional vascular planes were used to guide manual positioning of $\mathrm{PO}_{2}$ recordings on and along targeted vessels. Tissue $\mathrm{PO}_{2}$ measurements were at a depth $\sim 200$ to $300 \mu \mathrm{m}$ and were chosen close to arterioles. Vascular $\mathrm{PO}_{2}$ measurements were recorded from the surface until a depth $\sim 250 \mu \mathrm{m}$.

\subsection{Simulation of Oxygen Diffusion in Tissue}

The Krogh model ${ }^{17}$ was used to simulate the initial dip in tissue oxygen tension at different distances from the arterioles and compare with experimental data

$T_{X}=T_{0}-\frac{p}{K}$,

where $T_{0}$ is the vessel oxygen tension (atm) and $T_{x}$ is the tissue oxygen tension at radial distance $x(\mathrm{~cm})$ from the vessel center.
The rate of oxygen consumption $p$ is equivalent to the product of the cerebral metabolic rate of oxygen $\left(\mathrm{CMRO}_{2}\right)$ and the density $\rho$ of brain tissue. The Krogh diffusion constant $K$ is the product of the oxygen diffusion coefficient $D\left(1.5 \times 10 \mu \mathrm{m}^{2} / \mathrm{s}\right)$ and the oxygen solubility $\alpha\left[1.3 \times 10^{-3} \mathrm{~cm}^{3} /\left(\mathrm{cm}^{3} \times 760 \mathrm{mmHg}\right)\right]$. The Krogh model postulates that a central vessel with radius $r$ and infinite length supplies a concentric tissue cylinder with radius $R$ with radially diffusing oxygen. Using a quasisteady state approximation, the difference in $T_{x}$ obtained from above equation at baseline and during epilepsy gave an estimate of the initial dip versus distance from the vessel. For the mouse brain, the $\mathrm{CMRO}_{2}$ was assumed to be $2.6 \mu \mathrm{mol} / \mathrm{g} / \mathrm{min}$ at baseline $^{18}$ and to increase $\sim 12 \%$ during epileptic seizures. ${ }^{10}$ Measured changes in $T_{0}$ during seizures were used in the model.

To roughly model the undershoot-minima time versus distance from the vessel, the diffusion equation was solved for the same geometry with the initial conditions of baseline $\mathrm{CMRO}_{2}$ and $T_{0}$ assuming only $\mathrm{CMRO}_{2}$ changes during epilepsy. The time to reach the steady state was used as an estimate for the undershoot-minima time.

\section{Results}

\subsection{Oxygen Partial Pressure Changes in Tissue at the Epileptic Focus during Seizure-Like Activity}

Seizure-like activity was characterized by first rhythmic spiking of increasing amplitude and decreasing frequency, evolving into rhythmic spikes and slow wave activity prior to gradual offset [see e.g., Figs. 1(a) middle and 1(b) middle].

A typical change in tissue $\mathrm{PO}_{2}$ is shown in Fig 1(a). The $\mathrm{PO}_{2}$ time-profile was biphasic with an early dip after ictal onset, followed by a longer duration increase in $\mathrm{PO}_{2}$. These results were in agreement with previous works where similar responses were observed using confocal microscopy or oxygen electrodes. ${ }^{6,13}$
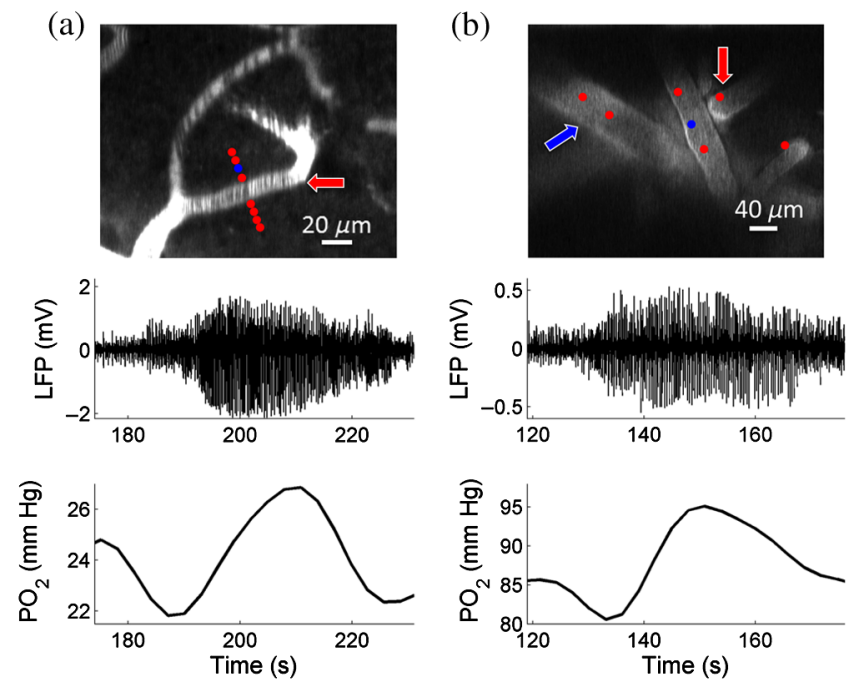

Fig. 1 Representative changes in $\mathrm{PO}_{2}$ in (a) tissue and (b) arteriole, in response to epileptic events in a local area. Grayscale angiogram of cortical pial tissue with points of interest [with arterioles shown by the red arrows, a venule shown by the blue arrow, and a typical $\mathrm{PO}_{2}$ time course shown for the blue points, top of (a) and (b) panels]. The time course of LFPs indicates seizure initiation. (a) Epileptic activity induced a transient dip in tissue $\mathrm{PO}_{2}$ followed by an increase in $\mathrm{PO}_{2}$ at the focus. (b) In the arteriole, the $\mathrm{PO}_{2}$ profile was also biphasic with an early dip followed by an increase. 

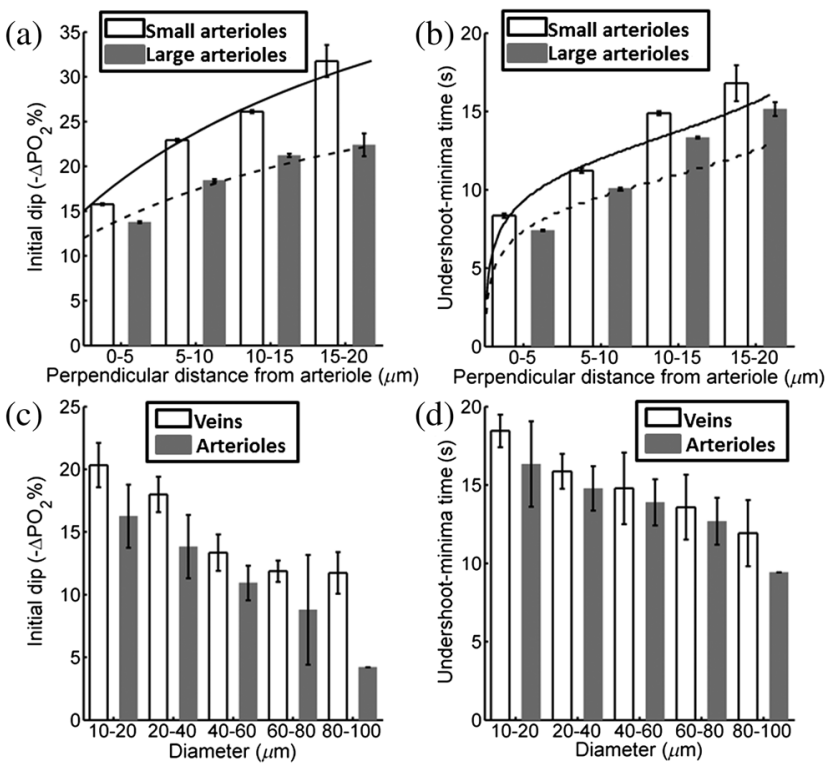

Fig. 2 (a) Changes in the amplitude of the percent initial dip around small and large arterioles, as a function of the perpendicular distance. The solid line and dashed line show simulated results with small and large arterioles separately. Error bars represent standard error of the mean (SEM). (b) Timing of the dip extrema as a function of arteriolar size and perpendicular distance with simulated results. (c) Percent change of the initial dip in arterioles and venules in response to seizure-like activity, grouped by vessel diameter, with corresponding standard error reported. (d) The undershoot-minima time in arterioles and venules, grouped by vessel diameter, with corresponding SEM.

We then investigated the spatial distribution of this dip along small arterioles. Four mice were used to measure the $\mathrm{PO}_{2}$ in tissue at multiple locations near an arteriole $(\sim 16$ arterioles per mouse) that was located in the epileptic focus, i.e., $<1.5 \mathrm{~mm}$ from the 4-AP injection site. Figure 2(a) shows that the percent $\mathrm{PO}_{2}$ changes during the dip were significantly lower in tissue closer to arterioles than that located father away.

This change was vessel size dependent as seen by separating data according to diameter in two groups, $12.60 \pm 0.83 \mu \mathrm{m}$ $(n=38$, mean $\pm \mathrm{SD})$ and $14.65 \pm 1.05 \mu \mathrm{m}(n=25)$ with significantly larger changes in tissue surrounding smaller arterioles ( $t$-test, $p<0.001)$.

The undershoot-minima time [Fig. 2(b)] was also seen to be lower when the point was located closer to the arteriole or next to a larger vessel ( $t$-test, $p=0.005$ ). Both trends of initial dip and undershoot-minima time could be predicted from simulations with the Krogh-Erlang model and diffusion equation [lines in Figs. 2(a) and 2(b)].

\subsection{Epileptic Seizures-Induced Oxygen Partial Pressure Response in Vasculature at the Epileptic Focus}

Since smaller arterioles are likely to be located further downstream than larger ones, we investigated $\mathrm{PO}_{2}$ changes in upstream and downstream vascular segments during similar ictal events. Four mice were used to gather recordings at multiple locations in arterioles and venules at the epileptic focus $(<1.5 \mathrm{~mm}$ from the injection site). Figures 2(c) and 2(d) show the percent changes associated with the initial dip and undershoot-minima time in arterioles and venules categorized by diameter. Figure 2(c) shows a size-dependent initial dip with smaller vessels seeing larger changes. Moreover, the observed variations in $\mathrm{PO}_{2}$ were larger in venules than arterioles ( $t$-test, $p=0.03$ ). Figure 2(d) further indicates that the undershootminima time of large arterioles and venules occurred earlier than small arterioles and venules. There was also a significant difference between the undershoot-minima time values of the arterioles and venules ( $t$-test, $p=0.01$ ).

\section{Discussion}

Epilepsy-evoked changes in cerebral tissue oxygenation have been observed previously by Clark microelectrodes ${ }^{9}$ and confocal phosphorescence lifetime microscopy. ${ }^{13}$ Polarographic electrode oxygen measurements have limited spatial accuracy and are limited in the number of point measurements achievable. In our previous work, confocal phosphorescence lifetime microscopy was also limited due to its inability to reach deeper tissue making investigations of the impact of microvascular morphology on delivery difficult. To our knowledge, the current study is the first to perform absolute measurements of $\mathrm{PO}_{2}$ in multiple individual microvascular compartments and tissue locations with high spatial and temporal resolution during epileptic seizures.

\subsection{Relationship between the Initial Dip and Distance from Arterioles}

Exploiting spatial measures around small arterioles, we investigated how tissue oxygen pressure changed near arterioles located in the epileptic focus region during epileptiform activity. Our data indicate a decreased $\mathrm{PO}_{2}$ percent change surrounding small arterioles during early phases of epileptic activity (initial dip), which can be attributed to increased tissue metabolic consumption using a Krogh-Erlang model. While capillary delivery may increase its contribution, in these events they do not fully compensate for consumption needs with small and large arterioles remaining largely responsible for oxygen distribution in the cortex according to modeling. ${ }^{19}$ This is consistent with previous findings of oxygen exchange between large arterioles and tissue. ${ }^{13}$ Our results further suggest that it is tissue areas located far from larger arterioles, in the capillary bed, which are more susceptible to hypoxia rather than tissue next to arterioles. Temporally, our data show that points far away from the arteriolar wall take more time to recover basal $\mathrm{PO}_{2}$ than locations near small arterioles with a size-dependent arteriolar influence. The observed delay times are larger than typical blood transit time $(\sim 2 \mathrm{~s})$ in the neurovascular unit. Overall these results suggest that the increased $\mathrm{CBF}$ and cerebral blood volume in the focus during epileptic events will supply more oxygen to the tissue near arterioles but may not always meet the demands of oxygen metabolism in capillary areas. An overshoot in CBF might be required to supply these areas adequately to avoid hypoxia.

\subsection{Relationship between the Initial Dip and Diameter of Arterioles and Venules}

The largest fractional decreases in vascular $\mathrm{PO}_{2}$ were measured in small venules and small arterioles. As upstream vessels deliver more oxygen during intense neural activity, downstream and smaller vessels see decrease both due to upstream consumption and local tissue need. The lower dip observed in large venules also suggest the presence of short vascular paths between arterioles and venules or shunts. 


\section{Neurophotonics Letters}

\section{Conclusion}

This study provided absolute $\mathrm{PO}_{2}$ measurements in tissue, arterioles, and venules with TPM during 4-AP evoked epileptic events and observed vascular architecture dependent changes of $\mathrm{PO}_{2}$ delivery.

\section{Disclosures}

Dr. Lesage reports a minority ownership in Labeo Technologies Inc.

\section{Acknowledgments}

We thank Marc-Antoine Gillis and Natacha Duquette for their assistance in animal preparation. We also thank Sergei Vinogradov for calibrating the PtP-C343 dye for us. We also acknowledge support from the Canadian Institutes of Health Research under Grant No. 299166 to F. Lesage and from the National Institutes of Health under Grant No. R24-NS092986.

\section{References}

1. H. M. Duvernoy, S. Delon, and J. L. Vannson, "Cortical blood vessels of the human brain," Brain Res. Bull. 7, 519-579 (1981).

2. F. Lauwers et al., "Morphometry of the human cerebral cortex microcirculation: general characteristics and space-related profiles," NeuroImage 39, 936-948 (2008).

3. T. Dalkara and L. Alarcon-Martinez, "Cerebral microvascular pericytes and neurogliovascular signaling in health and disease," Brain Res. 1623, 3-17 (2015).

4. X. Hu and E. Yacoub, "The story of the initial dip in fMRI," NeuroImage 62, 1103-1108 (2012).

5. P. Pouliot et al., "Tight neurovascular coupling in a rat model of quasiperiodic interictal spiking using multispectral optical imaging," Proc. SPIE 8412, 841203 (2012).
6. S. Bahar et al., "Intrinsic optical signal imaging of neocortical seizures: the 'epileptic dip"," Neuroreport 17, 499-503 (2006).

7. R. B. Buxton and L. R. Frank, "A model for the coupling between cerebral blood flow and oxygen metabolism during neural stimulation," J. Cereb. Blood Flow Metab. 17, 64-72 (1997).

8. Y. Zheng et al., "A model of the hemodynamic response and oxygen delivery to brain," Neurolmage 16, 617-637 (2002).

9. M. Zhao et al., "Focal increases in perfusion and decreases in hemoglobin oxygenation precede seizure onset in spontaneous human epilepsy," Epilepsia 48, 2059-2067 (2007).

10. M. Zhao et al., "Preictal and ictal neurovascular and metabolic coupling surrounding a seizure focus," J. Neurosci. 31, 13292-13300 (2011).

11. A. S. Geneslaw et al., "Tissue hypoxia correlates with intensity of interictal spikes," J. Cereb. Blood Flow Metab. 31, 1394-1402 (2011).

12. M. Zhao et al., "Spatiotemporal dynamics of perfusion and oximetry during ictal discharges in the rat neocortex," J. Neurosci. 29, 28142823 (2009).

13. C. Zhang et al., "Measurement of local partial pressure of oxygen in the brain tissue under normoxia and epilepsy with phosphorescence lifetime microscopy," PLoS One 10, e0135536 (2015).

14. S. Sakadžić et al., "Large arteriolar component of oxygen delivery implies a safe margin of oxygen supply to cerebral tissue," Nat. Commun. 5, 5734 (2014).

15. O. S. Finikova et al., "Oxygen microscopy by two-photon-excited phosphorescence," ChemPhysChem 9, 1673-1679 (2008).

16. J. Lecoq et al., "Simultaneous two-photon imaging of oxygen and blood flow in deep cerebral vessels," Nat. Med. 17, 893-898 (2011).

17. A. Krogh, "The number and distribution of capillaries in muscles with calculations of the oxygen pressure head necessary for supplying the tissue," J. Physiol. 52, 409-415 (1919).

18. W. Cui et al., "Non-invasive measurement of cerebral oxygen metabolism in the mouse brain by ultra-high field ${ }^{17} \mathrm{O}$ MR spectroscopy," J. Cereb. Blood Flow Metab. 33, 1846-1849 (2013).

19. M. Sharan et al., "Experimental and theoretical studies of oxygen gradients in rat pial microvessels," J. Cereb. Blood Flow Metab. 28, 1597 1604 (2008). 